Prepared for the U.S. Department of Energy under Contract DE-AC05-76RL01830

\title{
Analytical Data Report for Samples Collected From 100-N Area Aquifer Tubes, Sample Delivery Group ESL080015
}

Michael Lindberg

September 2008

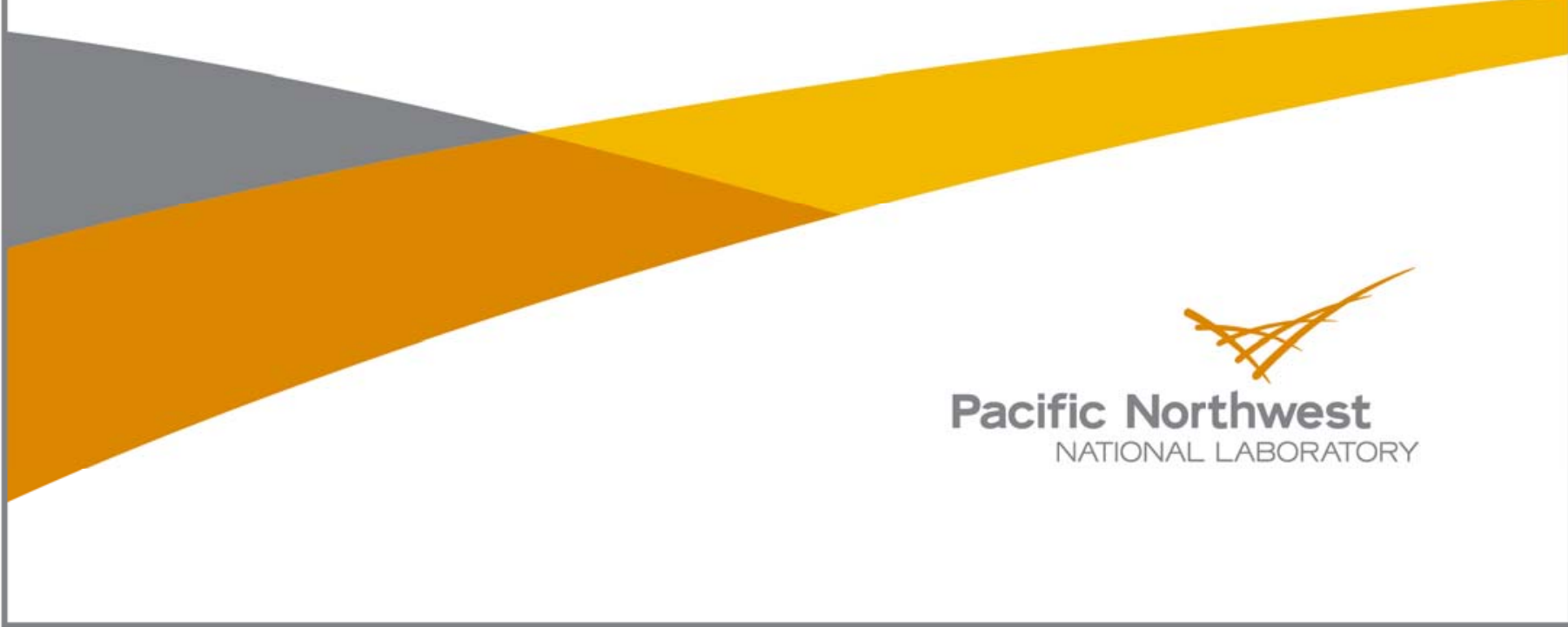


09/28/08 08:53

\section{To: Dana Widrig}

From: Michael J. Lindberg

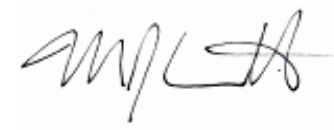

Environmental Sciences Laboratory

Energy and Environment Directorate, Pacific Northwest National Laboratory

Subject: Analytical Data Report for Samples Collected From 100-N Area Aquifer Tubes, Sample Delivery Group ESL080015, SAF Number G08-003

This letter contains the following information for sample delivery group ESL080015

- Cover Sheet

- Narrative

- Analytical Results

- Chain of Custodies 


\section{Introduction}

On March 31, 2008 water samples were received from 100-N Area Aquifer Tubes for geochemical studies.

\section{Analytical Results/Methodology}

The analyses for this project were performed at the 325 building located in the 300 Area of the Hanford Site. The analyses were performed according to Pacific Northwest National Laboratory (PNNL) approved procedures and/or nationally recognized test procedures. The data sets include the sample identification numbers, analytical results, estimated quantification limits (EQL), and quality control data.

\section{Quality Control}

The preparatory and analytical quality control requirements, calibration requirements, acceptance criteria, and failure actions are defined in the on-line QA plan “Conducting Analytical Work in Support of Regulatory Programs” (CAW). This QA plan implements the Hanford Analytical Services Quality Assurance Requirements Documents (HASQARD) for PNNL.

\section{Definitions}

Dup Duplicate

RPD Relative Percent Difference

\section{Sample Receipt}

Samples were received with a chain of custody (COC) and were analyzed according to the sample identification numbers supplied by the client. All Samples were refrigerated upon receipt until prepared for analysis.

All samples were received with custody seals intact unless noted in the Case Narrative.

\section{Holding Times}

Holding time is defined as the time from sample preparation to the time of analyses. The prescribed holding times were met for all analytes unless noted in the Case Narrative.

\section{Analytical Results}

All reported analytical results meet the requirements of the CAW or client specified SOW unless noted in the case narrative. 


\section{Case Narrative Report}

\section{DISCLAIMER}

This report was prepared as an account of work sponsored by an agency of the United States Government. Neither the United States Government nor any agency thereof, nor Battelle Memorial Institute, nor any of their employees, makes any warranty, express or implied, or assumes any legal liability or responsibility for the accuracy, completeness, or usefulness of any information, apparatus, product, or process disclosed, or represents that its use would not infringe privately owned rights. Reference herein to any specific commercial product, process, or service by trade name, trademark, manufacturer, or otherwise does not necessarily constitute or imply its endorsement, recommendation, or favoring by the United States Government or any agency thereof, or Battelle Memorial Institute. The views and opinions of authors expressed herein do not necessarily state or reflect those of the United States Government or any agency thereof.

\section{SAMPLES INCLUDED IN THIS REPORT}

\section{Fluor Citrate}

$\begin{array}{lll}\text { HEIS No. } & \text { Laboratory ID } & \text { Matrix } \\ \text { B1THL4 } & 0803028-01 & \text { WATER } \\ \text { B1THL9 } & 0803028-02 & \text { WATER } \\ \text { B1THK4 } & 0803028-03 & \text { WATER } \\ \text { B1THK8 } & 0803028-04 & \text { WATER } \\ \text { B1THM4 } & 0803028-05 & \text { WATER } \\ \text { B1THM7 } & 0803028-06 & \text { WATER }\end{array}$

\begin{tabular}{llll} 
Date Collected & \multicolumn{2}{l}{ Date Received } \\
3/25/08 & $10: 33$ & $3 / 31 / 08$ & $12: 45$ \\
3/25/08 & $11: 22$ & $3 / 31 / 08$ & $12: 45$ \\
3/25/08 & $09: 01$ & $3 / 31 / 08$ & $12: 45$ \\
3/25/08 & $09: 34$ & $3 / 31 / 08$ & $12: 45$ \\
3/25/08 & $12: 03$ & $3 / 31 / 08$ & $12: 45$ \\
3/26/08 & $08: 22$ & $3 / 31 / 08$ & $12: 45$
\end{tabular}


The following analyses were performed on the following samples included in this report:

Anions By Ion Chromatography

\section{SAMPLES ANALYZED IN THIS REPORT}

$\begin{array}{lll}\text { HEIS No. } & \text { Laboratory ID } & \text { Matrix } \\ \text { B1THL4 } & 0803028-01 & \text { WATER } \\ \text { B1THL9 } & 0803028-02 & \text { WATER } \\ \text { B1THK4 } & 0803028-03 & \text { WATER } \\ \text { B1THK8 } & 0803028-04 & \text { WATER } \\ \text { B1THM4 } & 0803028-05 & \text { WATER } \\ \text { B1THM7 } & 0803028-06 & \text { WATER }\end{array}$

\begin{tabular}{llll} 
Date Collected & \multicolumn{2}{c}{ Date Received } \\
3/25/08 & $10: 33$ & $3 / 31 / 08$ & $12: 45$ \\
3/25/08 & $11: 22$ & $3 / 31 / 08$ & $12: 45$ \\
3/25/08 & $09: 01$ & $3 / 31 / 08$ & $12: 45$ \\
$3 / 25 / 08$ & $09: 34$ & $3 / 31 / 08$ & $12: 45$ \\
3/25/08 & $12: 03$ & $3 / 31 / 08$ & $12: 45$ \\
$3 / 26 / 08$ & $08: 22$ & $3 / 31 / 08$ & $12: 45$
\end{tabular}




\section{Anions by Ion Chromatography}

\begin{tabular}{|c|c|c|c|c|c|c|c|}
\hline CAS \# & Analyte & Results & Units & EQL & Analyzed & Batch & Method \\
\hline HEIS No. & B1THL4 & \multicolumn{2}{|c|}{ Lab ID: } & 0803028-01 & & & \\
\hline $126-44-3$ & Citrate & $<1.00 \mathrm{E}-1$ & $\mathrm{ug} / \mathrm{mL}$ & $1.00 \mathrm{E}-1$ & $4 / 08 / 08$ & 8D08003 & AGG-IC-001 \\
\hline HEIS No. & B1THL9 & \multicolumn{2}{|c|}{ Lab ID: } & 0803028-02 & & & \\
\hline $126-44-3$ & Citrate & $<1.00 \mathrm{E}-1$ & $\mathrm{ug} / \mathrm{mL}$ & 1.00E-1 & $4 / 08 / 08$ & 8D08003 & AGG-IC-001 \\
\hline HEIS No. & В1THK4 & \multicolumn{2}{|c|}{ Lab ID: } & 0803028-03 & & & \\
\hline $126-44-3$ & Citrate & $<1.00 \mathrm{E}-1$ & $\mathrm{ug} / \mathrm{mL}$ & $1.00 \mathrm{E}-1$ & 4/08/08 & 8D08003 & AGG-IC-001 \\
\hline HEIS No. & В1ТНК8 & \multicolumn{2}{|c|}{ Lab ID: } & 0803028-04 & & & \\
\hline $126-44-3$ & Citrate & $<1.00 \mathrm{E}-1$ & $\mathrm{ug} / \mathrm{mL}$ & $1.00 \mathrm{E}-1$ & 4/08/08 & 8D08003 & AGG-IC-001 \\
\hline HEIS No. & B1THM4 & \multicolumn{2}{|c|}{ Lab ID: } & 0803028-05 & & & \\
\hline $126-44-3$ & Citrate & $<1.00 \mathrm{E}-1$ & $\mathrm{ug} / \mathrm{mL}$ & $1.00 \mathrm{E}-1$ & 4/08/08 & 8D08003 & AGG-IC-001 \\
\hline HEIS No. & B1THM7 & \multicolumn{2}{|c|}{ Lab ID: } & 0803028-06 & & & \\
\hline $126-44-3$ & Citrate & $<1.00 \mathrm{E}-1$ & $\mathrm{ug} / \mathrm{mL}$ & 1.00E-1 & $4 / 09 / 08$ & 8D08003 & AGG-IC-001 \\
\hline
\end{tabular}




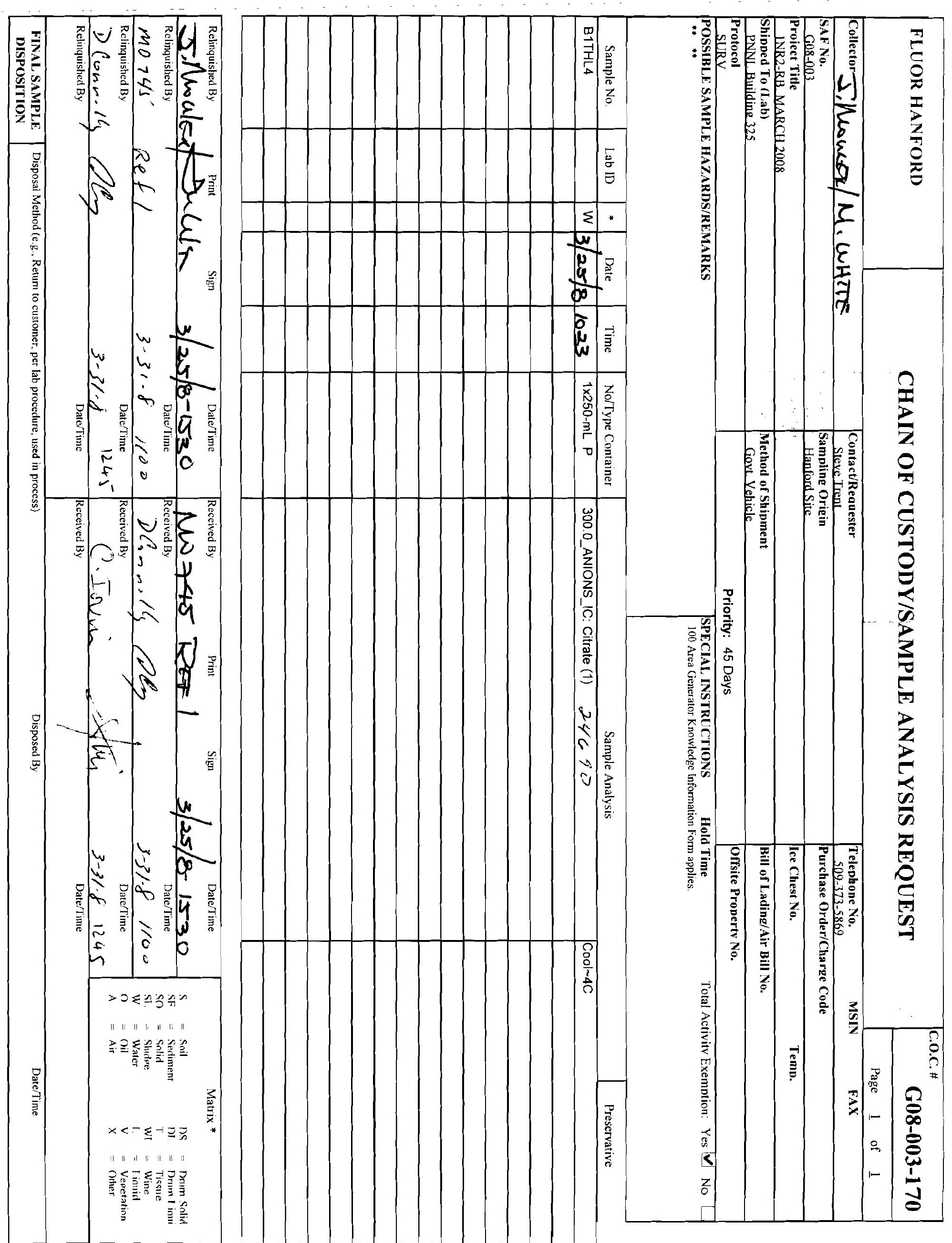




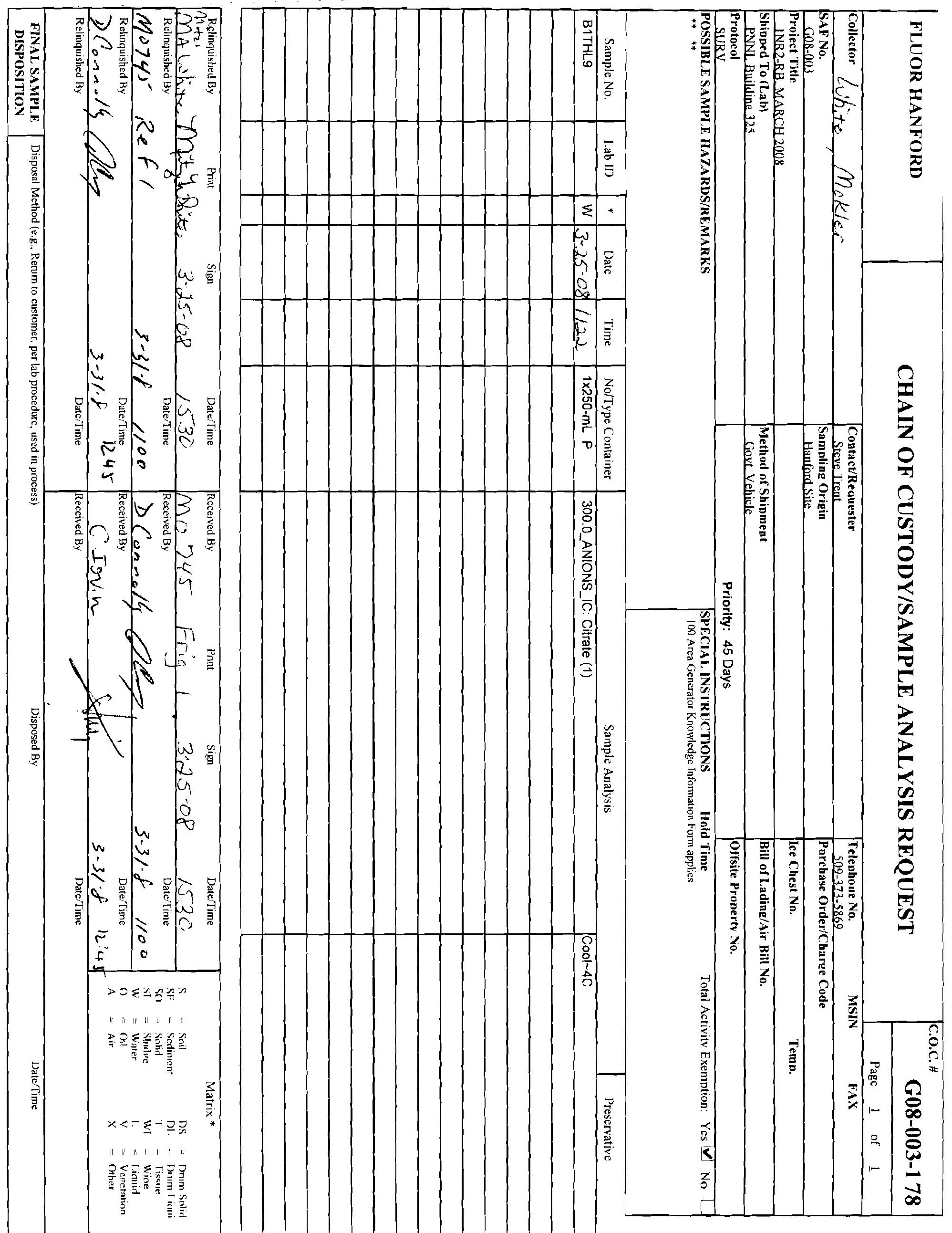




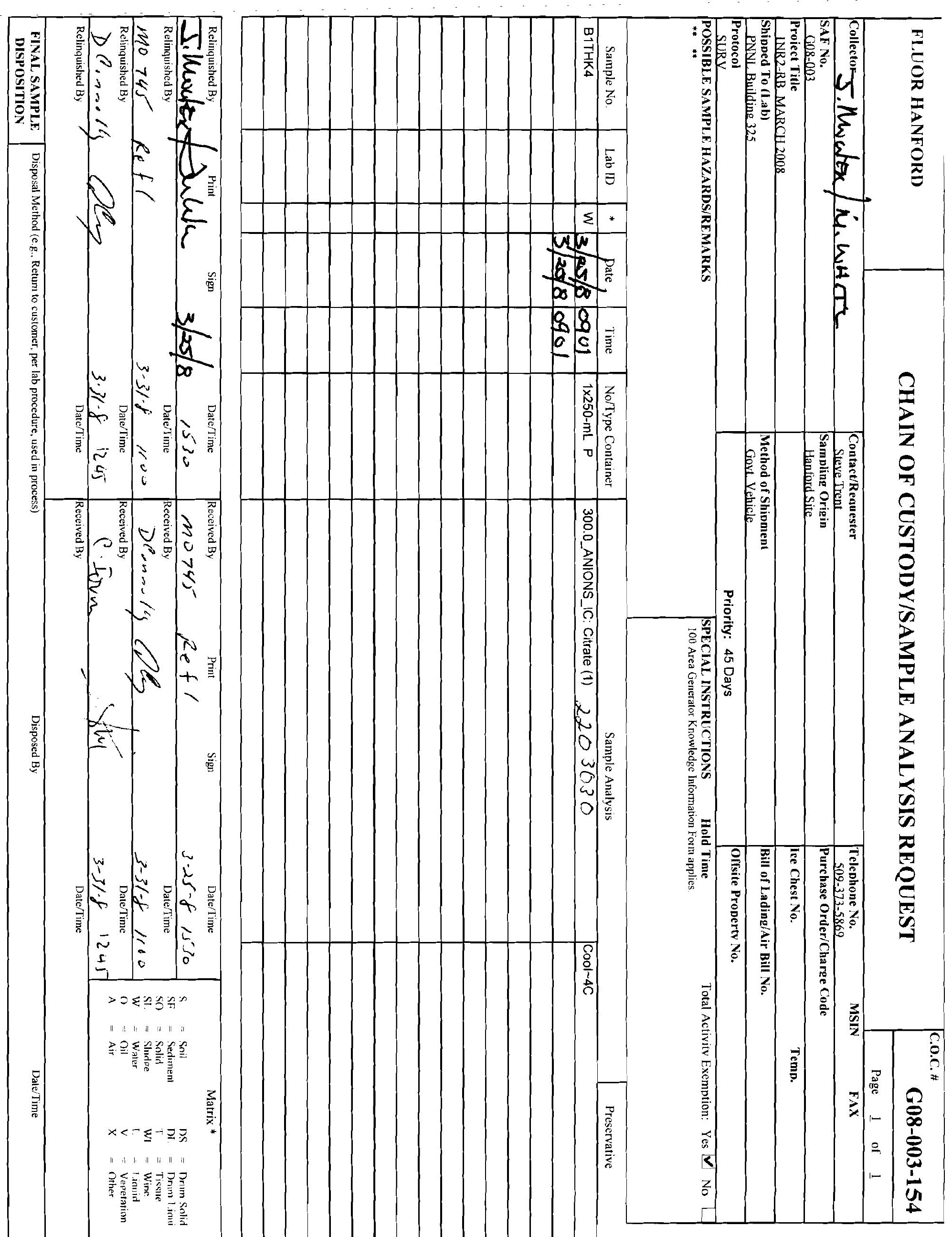



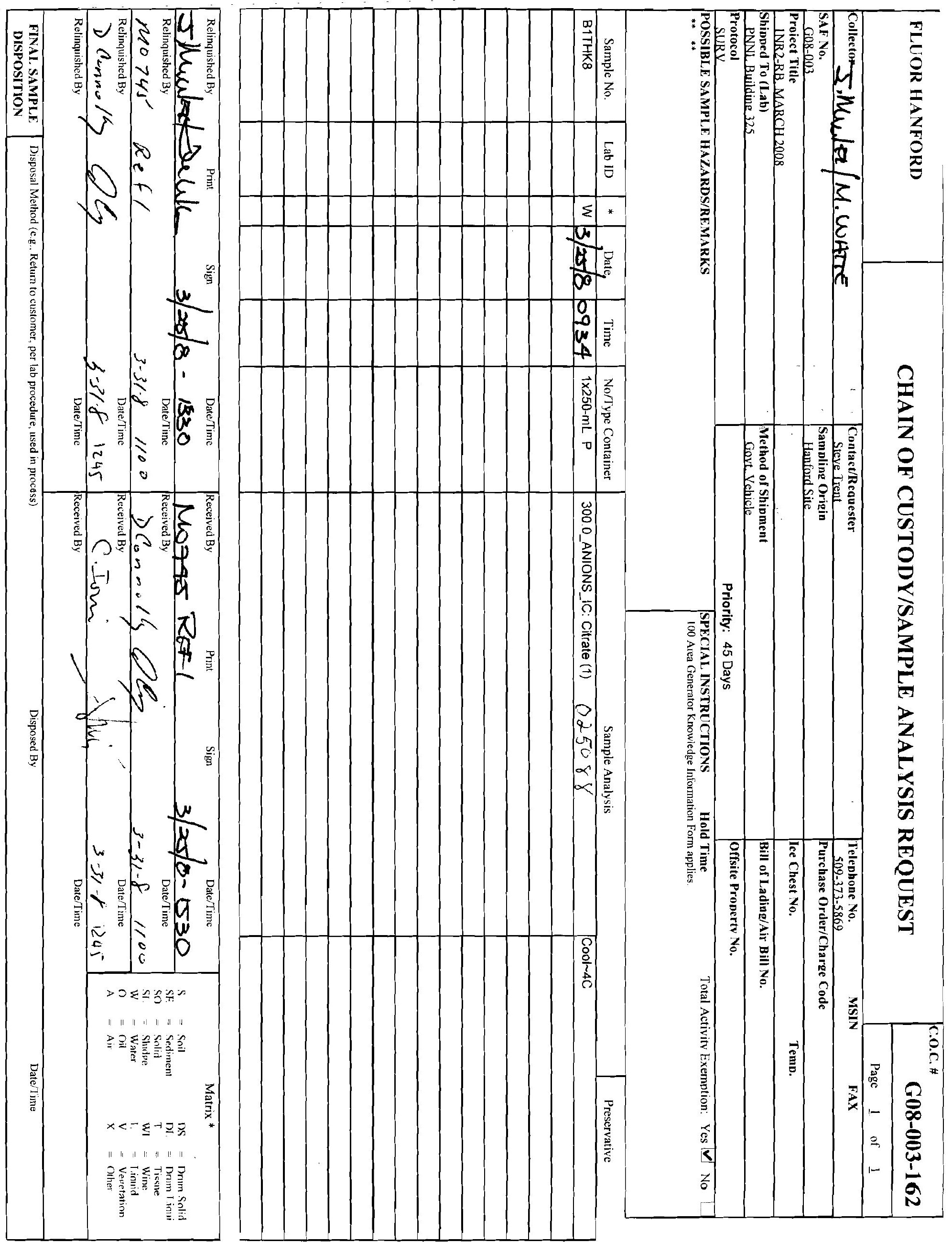


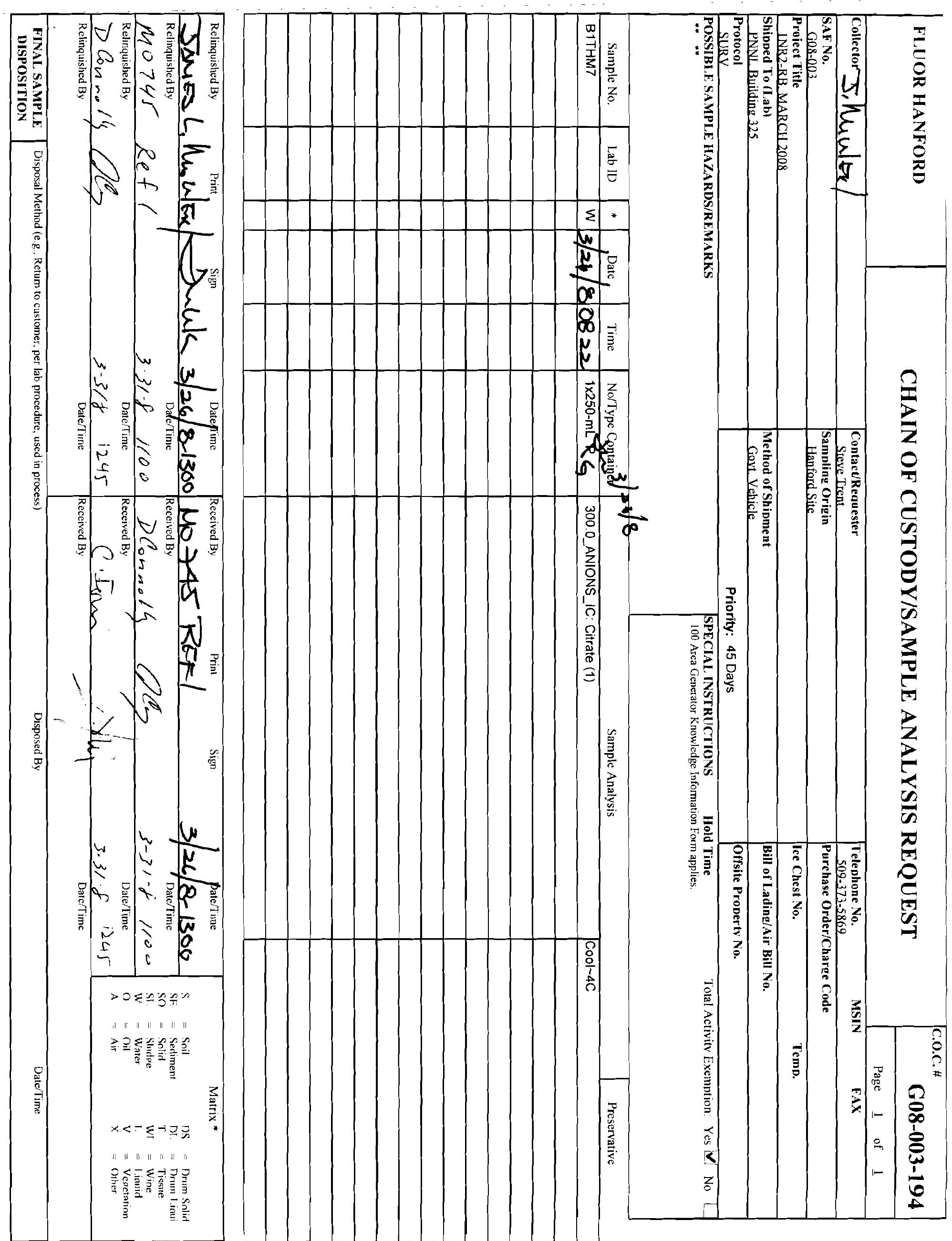

